\title{
Evaluation du bénéfice des médicaments hors étiquette
}

\author{
Dans le cadre des articles 71 a et 71 b de l'ordonnance sur l'assurance-maladie, le \\ Service du médecin-conseil d'Helsana a mis au point un modèle de notation qui, en \\ collaboration avec l'industrie pharmaceutique, permet un remboursement, par l'as- \\ sureur, adapté au bénéfice du patient. Les critères d'évaluation et la concrétisation \\ seront présentés ci-après.
}

Beat Seiler ${ }^{a}$, Robert Fries ${ }^{b}$, Hanspeter Honegger ${ }^{c}$

a Dr méd., M.H.A. Chef médicin-conseil Helsana

b Dr méd., médecin-conseil Helsana

c Prof. Dr méd, ancien directeur médical de la clinique d'oncologie Stadtspital Triemli Zürich
Correspondance:

Dr Beat Seiler

Case postale

CH-8081 Zurich

Tél. 0433406224

beat.seiler[at]helsana.ch
Le principe selon lequel les médicaments ne sont remboursés que s'ils figurent sur la liste des spécialités (LS) et respectent l'indication enregistrée ou limitée s'applique dans l'assurance de base obligatoire (AOS). L'utilisation de médicaments en dehors de la LS, de médicaments uniquement autorisés à l'étranger ou de médicaments employés dans une autre limitation/ indication est devenue de plus en plus fréquente ces dernières années. Un fossé se creuse notamment en oncologie entre les exigences cliniques du médecin, les revendications du patient et le remboursement des médicaments, en raison de l'absence d'agrément de certains médicaments ou des restrictions qui leur sont imposées.

\section{Réglementation du remboursement selon les art. 71 a et $71 \mathrm{~b}$ OAMal}

Dans les deux articles $71 \mathrm{a}$ et $71 \mathrm{~b}$ de l'ordonnance sur l'assurance-maladie (OAMal), le Conseil fédéral a défini l'obligation de remboursement en dérogation à la règle au $1^{\text {er }}$ mars 2011. Lorsqu'un médicament est utilisé en dehors de la LS ou de façon générale en dehors de l'indication autorisée, un remboursement au titre de l'AOS n'est possible que si certains critères sont remplis:

- La situation est susceptible de mettre la vie de l'assuré en danger ou de lui causer des problèmes de santé graves et chroniques, ou le médicament fait partie d'un complexe thérapeutique.

- Faute d'alternative thérapeutique, il n'existe pas d'autre traitement efficace autorisé.

Le médicament doit en outre permettre d'escompter un bénéfice thérapeutique élevé.

La nouveauté réside dans le fait que le remboursement n'est plus constitué d'un montant fixe de la LS ou de l'industrie pharmaceutique, mais que les assureurs fixent le montant du remboursement en fonction du bénéfice escompté du médicament.

\section{Enjeux des art. 71 a et 71 b OAMal}

Afin de permettre à l'assureur de procéder à un remboursement adapté au bénéfice, le médecin-conseil doit dans un premier temps répondre à la question du bénéfice thérapeutique élevé, puis de l'adéquation progressive du bénéfice élevé comme base pour le montant du remboursement.

La difficulté provient de la nécessité de tirer des conclusions quant au bénéfice dans un délai souvent très court en s'appuyant sur un nombre limité de données. Des décisions relatives à la prestation d'assurance dans le cas particulier et dans le contexte de la maladie doivent en outre être prises, sans oublier que l'état des études change souvent en l'espace de quelques mois. Des ajustements rapides sont donc indispensables.

Des évaluations exhaustives assorties d'évaluations technologiques (HTA) [1] qui reposent sur des données très complètes et nécessitent des mois ne sont pas adaptées dans ce domaine.

\section{Concept du nouveau modèle de bénéfice d'Helsana}

Le présent modèle d'évaluation du bénéfice repose sur une estimation du bénéfice sans évaluation comparative, conformément aux dispositions des art. 71 a et $71 \mathrm{~b}$ OAMal. Une notation des médicaments pour lesquels il n'existe pas d'alternatives efficaces et autorisées est réalisée. Le modèle de notation est en outre utilisé pour des affections graves, chroniques, voire mortelles ou pour un usage dans le cadre de complexes thérapeutiques.

La priorité a été donnée à la transparence quant à la définition d'une évaluation scientifiquement fondée du bénéfice et des coûts d'un médicament concret, dans le cadre de la mise en place et du développe-

\section{Tableau 1}

Concept du modèle d'un Service du médecin-conseil (SMC):

- Possède un barème d'évaluation qui autorise une évaluation aussi objective et compréhensible que possible, focalisée sur le «bénéfice thérapeutique» des études et des situations des patients;

- Possède un score permettant de décrire un échelonnement progressif du bénéfice thérapeutique;

- Donne une indication rapide et d'actualité sur le bénéfice médico-thérapeutique. 
ment. Afin de garantir son utilisation opérationnelle au quotidien, la notation se fonde sur des critères clairement définis et compréhensibles par tous. L'évaluation doit être aussi objective et logique que possible et autoriser des conclusions pertinentes concernant l'importance et l'échelonnement du bénéfice thérapeutique (tabl. 1).

Le concept est organisé suivant les principes de la médecine fondée sur des données probantes (EBM) [2]. Le choix des critères et leur pondération sont scientifiquement étayés. Les travaux fondamentaux d'Ocana et Tannock [3], de Bhattacharya [4] et de Simon [5] se sont notamment avérés essentiels, tout comme les critères d'évaluation de la FDA [6]. De nombreux travaux spécifiques sur la question de l'évaluation du bénéfice, par ex. ceux d'Amir [7], le modèle en collaboration avec le professeur Hanspeter Honegger, qui a longtemps été médecin-chef du service d'oncologie/hématologie à l'hôpital Triemli à Zurich. Grâce à l'expérience du professeur Honegger, le système de scoring inclut la base scientifique, mais aussi un élément pratique, qui est indispensable dans la perspective du «bénéfice thérapeutique» (tabl. 2).

Le temps nécessaire à une évaluation du bénéfice est de 30 à 60 minutes, si les données scientifiques et le rapport clinique sont disponibles. Des informations détaillées sur la notation, y compris les tableaux de scores, ont été rassemblées dans l'ouvrage publié par les éditions de la Société suisse pour la politique de la santé (SSPS) [10].

S'agissant des maladies rares ou de situations où les traitements n'ont pas produit de résultats, le

\section{«L'évaluation du rapport coûts/utilité ne portant pas uniquement sur des questions médicales, mais aussi toujours sur des questions norma- tives, un consensus social est incontournable.»}

Baker [8] et Fayers [9] ont cependant également été pris en compte. Grâce à la prise en compte de la situation du patient, le modèle devient un instrument de l'évaluation du cas particulier au quotidien.

L'une des forces du concept réside dans le fait qu'aucune connaissance technique spécifique n'est requise pour l'évaluation. L'étude (randomisée) actuellement la plus importante et la plus pertinente doit être présentée au médecin-conseil par l'expert. Chaque médecin qui se penche sur une étude peut également procéder à une notation et tirer des conclusions quant au bénéfice.

\section{Le système de scoring pour la détermination du bénéfice}

Le Service du médecin-conseil d'Helsana a élaboré

Tableau 2

Critères d'évaluation:

$\begin{array}{ll}\text { Modèle SMC } & \text { Critères pour l'évaluation } \\ \text { Pertinence de l'étude } & \text { - Mécanisme d'action (Target) } \\ & \text { - Données probantes de l'étude concernant l'efficacité } \\ \text { - considérable } & \text { - Overall survival (OS) et Hazard Ratio (HR) } \\ \text { - significative } & \text { - Progression Free Survival (PFS)/Progression Free Rate (PFR) et HR } \\ \text { - faible } & \text { - Response Rate (RR) } \\ \text { - aucune } & \text { - Valeur p } \\ & \text { - Confiance dans l'étude } \\ \text { Concordance } & \text { - Dépenses concomitantes, qualité de vie } \\ \text { de l'étude patient- } & \text { - Concordance clinique } \\ \text { médicament } & \text { - Concordance médicament } \\ \text { - élevée } & \\ \text { - moyenne clinique } \\ \text { - aucune }\end{array}$

groupe de travail avait conscience du fait que des médicaments étaient souvent utilisés pour lesquels il n'existe aucune donnée d'études dans ces indications particulières susceptibles de satisfaire aux critères scientifiques requis pour démontrer l'efficacité d'un médicament. Dans leur indication, les médecins traitants se fondent sur des résumés de rapports de congrès, des rapports d'enquête ou des analogies plausibles entre principes actifs.

L'évaluation de telles données ne génère d'ailleurs que quelques points de scoring dans la plupart des cas (catégorie C, cf. la fig. 1). La preuve du bénéfice thérapeutique élevé exigé par l'art. 71 OAMal ne peut pas être apportée à la date de l'évaluation et le médecinconseil doit recommander le refus de la prise en charge des coûts. Pour que les patients concernés aient néanmoins la possibilité de suivre un traitement peut-être innovant et efficace, nous recommandons un essai thérapeutique dans de telles situations et nous conseillons un remboursement Pay-for-Performance à l'assureur. Il s'agit toutefois de cas isolés. La plupart des vérifications du bénéfice révèlent un bénéfice thérapeutique élevé et avéré (catégories A et B).

\section{Modèle SMC}

Le modèle distingue les champs d'action «Pharma» avec l'évaluation des études, «Médecin-conseil» avec l'adaptation du bénéfice à partir de l'évaluation du cas particulier et «Assureur» avec la définition du remboursement (fig. 1).

Modèles d'évaluation du rapport coûts/utilité L'analyse du rapport coûts/utilité est une méthode économique standard reconnue. L'évaluation de l'utilité est obtenue en déterminant la propension individuelle des citoyens à payer pour une prestation. 
Figure 1

Evaluation du bénéfice et remboursement des coûts.

Bénéfices et participation aux coûts

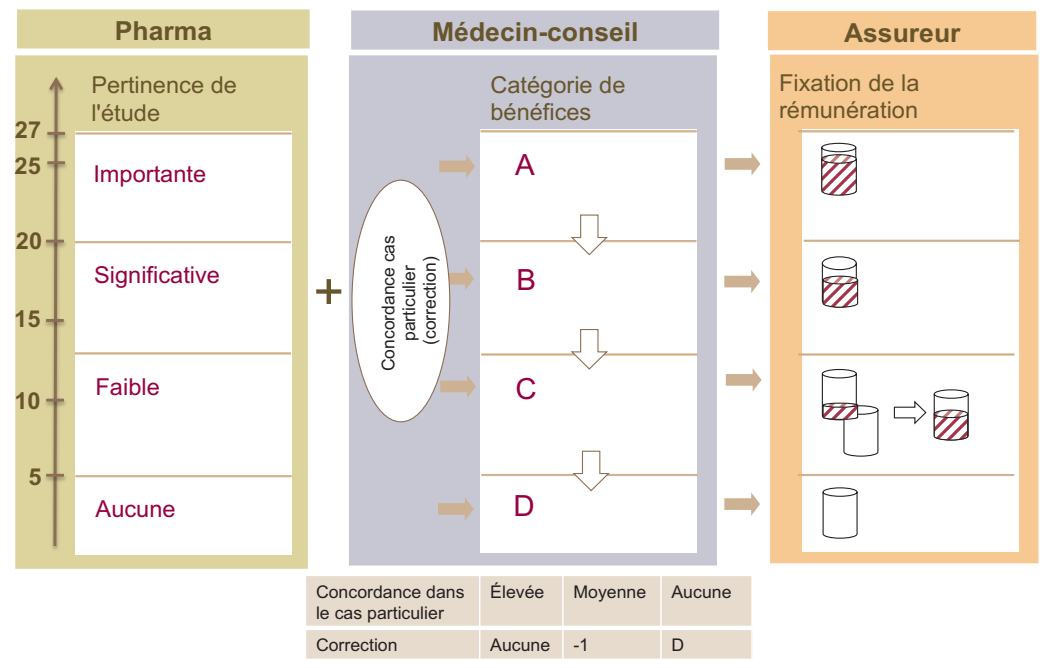

L'analyse du rapport coûts/valeur d'usage constitue une autre approche. Dans ce cas, la valeur d'usage se limite dans un premier temps à la qualité et à la durée de vie. Les «années de vie ajustées sur la qualité» (QALY pour Quality-Adjusted Life Years) sont la mesure la plus connue de l'utilité. Des valeurs-seuils ont ensuite été définies pour l'intégration dans le catalogue des prestations. Ainsi, le National Institute for Health Service (NICE) en Grande-Bretagne déconseille-t-il en général l'intégration si les coûts dépassent 30000 livres sterling.

Le modèle de J. Nadig [11] récemment publié dans le BMS associe l'évaluation de l'utilité et le remboursement par les caisses dans une application pragmatique du modèle de l'analyse coûts/utilité (propension à payer) et d'éléments de l'analyse du rapport coûts/valeur d'usage (qualité et durée de vie). Comme base du remboursement maximal, ce modèle définit le prix départ usine (PDU) de Glivec et de l'antagoniste du tryptophane $5 \mathrm{OH}$ et en déduit les coûts de recherche, de développement et de marketing. Il reste à savoir si ce modèle pourra être mis en œuvre et s'il sera accepté par les assureurs, les patients, les médecinsconseil et l'industrie pharmaceutique.

\section{Coopération entre l'industrie} pharmaceutique et les assureurs

Le patient doit pouvoir accéder rapidement à des médicaments innovants à l'efficacité évidente, à un coût raisonnable. Cet objectif peut être atteint grâce à la coopération entre les assureurs et les sociétés pharmaceutiques.

Les modèles de contrats, tels ceux conclus par Helsana avec Roche et Novartis, montrent la voie à suivre pour une utilisation de médicaments innovants dictée par la concurrence. En vertu des art. 71 a et $71 \mathrm{~b}$ OAMal, l'assureur a la possibilité de définir le remboursement des coûts à l'aune du bénéfice. Ces modèles autorisent une mise précoce sur le marché et récompensent avant tout les innovations.

\section{Une médecine personnalisée requiert de nouvelles approches}

La tendance dans le domaine de la «médecine personnalisée» requiert de nouvelles approches concernant l'évaluation du bénéfice et le remboursement des coûts. Des modifications de la pratique existante dans le domaine de la réglementation et des autorisations des médicaments ainsi que de nouvelles idées d'incitations seront nécessaires à l'avenir afin d'imposer le remboursement fondé sur des données probantes. Il est impératif de disposer d'informations fiables, générées par des études cliniques, sur l'amélioration de la qualité de vie susceptible de résulter de l'utilisation de ces médicaments.

L'évaluation du rapport coûts/utilité ne portant pas uniquement sur des questions médicales, mais aussi toujours sur des questions normatives, un consensus social est incontournable. A défaut, les décisions fondées sur une évaluation concrète du rapport coûts/ utilité auront beaucoup de mal à se faire accepter.

\section{Références}

1 Canadian Agency for Drugs and Technologies in Health HTA. Guidelines for the economic evaluation of health technologies: Canada. 3rd ed. Canadian Agency for Drugs and Technologies in Health (CADTH). Ottawa; 2006.

2 Centre for Evidence-Based Medicine. Levels of Evidence. Oxford. www.cebm.net/index.aspx?o=1025, 2001.

GRADE Working Group. Grading quality of evidence and strength of recomendations. BMJ 2004; 328 (7454):1490-4.

3 Ocana A, Tannock IF. When are «positive» clinical trials in Oncology truly positive? IJNI. 103:16-20.

4 Bhattacharya S, Fyfe G, Gray R, et al. Role of sensitivity analyses in assessing progression-free survival in late-stage oncology trials. JCO. 2009;27: 5958-64.

5 Simon R. Design and analysis of clinical trials. In: De Vita V, Lawrence T, Rosenberg S (ed.). Cancer, principles and practice of oncology; 2011. S. $720 \mathrm{ff}$.

6 Food and Drug Administration (FDA). Guidance for industry. Patient-reported outcome measures: Use in medical product development to support labeling claims.Draft guidance. 2006.

7 Amir E, Seruga B, Martinez-Lopez J, et al. Oncogenic targets, magnitude of benefit, and market pricing of antineoplastic drugs. JCO. 2011;29:2543-9.

8 Baker SG. Editorial: Surrogate Endpoints: Wishful Thinking or Reality. Journal of the National Cancer Institute. 2006;98:502-3.

9 Fayers P, Hays R. Assessing quality of life in clinical trials. Oxford: Oxford University Press; 2005.

10 Seiler B et al. Therapeutischer Nutzen eines Medikamentes: Bestimmung des therapeutischen Nutzens nach Art. $71 \mathrm{a} / \mathrm{b}$ der Verordnung zur Krankenversicherung KVV. SGGP. 2011;112.

11 Nadig J. Evidenzbasierte Nutzenbewertung: ein Model zur Vergütung des Off Label Use in der Onkologie. Bull Méd Suisses. 2012;93(14/15):552-5. 